\title{
A Combinational Optimization Approach for Advertising Budget Allocation
}

\author{
Deguang Kong, Xiannian Fan, Konstantin Shmakov and Jian Yang \\ Yahoo Research, Oath, 701 1st Ave, Sunnyvale, California, 94086 \\ \{dkong,xiannian.fan,kshmakov,jianyang\}@oath.com
}

\begin{abstract}
Bid optimization, which aims to find the competitive bid to achieve the best performance for the advertiser, is an important problem in online advertising. The optimal bid recommendation enables the advertisers to make informed decisions without actually spending the budget. In this paper, we consider a bid optimization scenario that the advertiser's budget can be split across multiple campaigns. To achieve the optimal performance, we formalize the bid optimization problem as a constraint combinational optimization problem, and derive an effective method to solve it. Experiment studies on realworld ad campaigns demonstrate the effectiveness of our method.
\end{abstract}

\section{CCS CONCEPTS}

\section{- Information systems $\rightarrow$ Computational advertising;}

\section{KEYWORDS}

Advertising; Optimization; Bidding

\section{ACM Reference Format:}

Deguang Kong, Xiannian Fan, Konstantin Shmakov and Jian Yang. 2018. A Combinational Optimization Approach for Advertising Budget Allocation. In WWW'18: The 2018 Web Conference Companion, April 23-27, 2018, Lyon, France. ACM, New York, NY, USA, 2 pages. https://doi.org/10.1145/3184558. 3186925

\section{INTRODUCTION}

Computational advertising (a.k.a online advertising) aims to serve the most relevant ads (from advertisers) matching to the particular context in web publisher. Computational advertising market has grown a lot and its market size will reach to over $\$ 250$ billion $^{1}$ in 2018. The publishers sell the ad inventories to the advertisers with the highest bidding via online auction mechanism in the nonguaranteed delivery of ads in sales channel. The ads market place acts as the platform for advertisers to bid for achieving the desired performance goals (such as impressions, clicks, etc). In computational ads, estimating the campaign performance at different bid prices will help the advertiser to plan their campaigns. In bid optimization, one important problem, which, however, has been ignored is that most advertisers have rigorous budget limits and they would like to split their budget across different campaigns in order to

\footnotetext{
${ }^{1}$ https://www.thecapitalgroup.com/us/insights/investment-insights/ tlv-digital.html
}

This paper is published under the Creative Commons Attribution 4.0 International (CC BY 4.0) license. Authors reserve their rights to disseminate the work on their personal and corporate Web sites with the appropriate attribution.

WWW'18, April 23-27, 2018, Lyon, France

() 2018 IW3C2 (International World Wide Web Conference Committee), published under Creative Commons CC BY 4.0 License.

ACM ISBN 978-1-4503-5640-4/18/04.

https://doi.org/10.1145/3184558.3186925 achieve the maximum return on investment (ROI). The question that naturally follows is: how to optimize the bid to maximize the KPIs (such as clicks, CTR [3]) given the budget limit shared by multiple campaigns?

To the best of our knowledge, this problem has not been well studied. Although there are some works that leverage supervised learning [7], second price auction theory ${ }^{2}$ and linear programming (LP) primal-dual method [2] for CPI optimization, and Markov Decision Process framework [1], inflection point [4] and CPA goal [5] for bid optimization, they are still quite limited in addressing the challenges: (C1) There are a broad margin of errors due to the realword data shift from estimation; (C2) There are very sparse and noisy observations for some campaigns; (C3) How to fairly and accurately split the budget to achieve the best KPI is non-trivial given the diversified campaigns.

To address these challenges, this paper presents the solutions that can automatically set bids for every advertiser to achieve the best performance goals across multiple campaigns given the fixed budget, which is actually similar to the process of choosing the proportions of budget be held in a portfolio, in such a way as to make the portfolio better than any others based on some criteria such as campaign KPI. However, the criteria for ad bid optimization is quite different from the expected value of the portfolio's rate of return as well as of the return's dispersion, possible risk and variance used in portfolio optimization [6] [8] in finance, which makes the traditional portfolio algorithm unable to work well here. Therefore, in this paper, we develop an effective algorithm to solve the portfolio optimization such that overall the advertiser can meet budget goals while the campaign achieves the best performance.

\section{METHODOLOGY}

Our goal is to find the best bidding price for each campaign that can maximize the overall KPI (e.g., clicks) at advertiser-level while satisfying the budget requirement. In the context of this paper, we use "maximum click" as the criterion for $\mathrm{KPI}^{3}$.

Suppose an advertiser initiated $k$ campaign cases and the overall advertiser budget is $B$, let the bidding price for campaign case $i$ be $b_{i}(1 \leq i \leq k)$, then the optimization goal is:

$$
\begin{aligned}
& \max _{b_{1}, b_{2}, \cdots, b_{k}} \sum_{i} \hat{h_{i}}\left(b_{i}, \theta^{i}\right) \\
& \text { s.t. } \sum_{i} \hat{g}_{i}\left(b_{i}, \Theta_{i}\right) \leq B, \quad b_{i} \geq 0,
\end{aligned}
$$

where $\hat{h_{i}}, \hat{g}_{i}$ denote estimated click and spend functions with parameter $\theta_{i}$ and $\Theta_{i}$ respectively. The obtained optimal solution

\footnotetext{
${ }^{2}$ https://en.wikipedia.org/wiki/Auction_theory

${ }^{3}$ The criterion of maximum cost-per-acquisition (CPA) or pay-peracquisition (PPA) can be similarly done.
} 
$\left(b_{1}^{*}, b_{2}^{*}, \cdots, b_{k}^{*}\right)$ is expected to maximize the overall clicks of the advertiser given the budget limit. To solve this problem, we propose an effective method that involves several steps:

- Step 1: Estimating the click and spend functions (with parameters $\theta_{i}$ and $\Theta_{i}$ ) using non-linear regression;

- Step 2: Solving the optimization using the constraint optimization method.

Let $\mathbf{b}=\left[b_{1}, b_{2}, \cdots, b_{k}\right]$, and

$$
H(\mathbf{b}):=\sum_{i} h_{i}\left(b_{i} ; \theta^{i}\right), \quad G(\mathbf{b}):=\sum_{i} g_{i}\left(b_{i} ; \Theta^{i}\right) .
$$

Notice that the Lagrangian function is:

$$
\begin{aligned}
& L(\mathbf{b}, \lambda, \mu)=H(\mathbf{b})+\lambda(G(\mathbf{b})-B)+\frac{\mu}{2}(G(\mathbf{b})-B)^{2}, \\
& \text { s.t. } \mathbf{b} \geq 0 .
\end{aligned}
$$

When optimizing $b_{i}$, the Lagrangian function concerning $b_{i}$ becomes:

$$
\begin{aligned}
& L\left(b_{i}, \lambda, \mu\right)=-h_{i}\left(b_{i}\right)+\lambda\left(g_{i}\left(b_{i}\right)+\sum_{j \neq i} g_{j}\left(b_{j}\right)-B\right) \\
& +\frac{\mu}{2}\left(g_{i}\left(b_{i}\right)+\sum_{j \neq i} g_{j}\left(b_{j}\right)-B\right)^{2}, \\
& \text { s.t. } b_{i} \geq 0 .
\end{aligned}
$$

Notice that

$$
\frac{\partial L\left(b_{i}, \lambda, \mu\right)}{\partial b_{i}}=-\frac{\partial h_{i}\left(b_{i}\right)}{\partial b_{i}}+\lambda \frac{\partial g_{i}\left(b_{i}\right)}{\partial b_{i}}+\mu\left(g_{i}\left(b_{i}\right)-D\right) \frac{\partial g_{i}\left(b_{i}\right)}{\partial b_{i}},
$$

where

$$
D=B-\sum_{j \neq i} g_{j}\left(b_{j}\right) .
$$

According to Karush-Kuhn-Tucker (KKT) condition, we have

$$
\begin{aligned}
& \lambda^{*} \geq 0, \quad \lambda^{*}(G(\mathbf{b})-B)=0, \\
& (G(\mathbf{b})-B) \leq 0, \quad \mathbf{b}_{\mathbf{i}}^{*} \geq \mathbf{0}, \quad \mu^{*}>\mathbf{0}, \\
& \frac{\partial L\left(b_{i}\right)}{\partial b_{i}^{*}} b_{i}^{*}=0(\forall i) .
\end{aligned}
$$

This gives the algorithm to solve $b_{i}$ using projection into nonnegative space, i.e.,

$$
b_{i} \leftarrow b_{i}-\eta \frac{\partial L\left(b_{i}\right)}{\partial b_{i}},
$$

where $\eta$ is the stepsize. The algorithm converges to the optimal solution.

\section{EXPERIMENT}

Given the accurate estimation of spend and clicks, as is observed in experimental study on multiple ads campaigns from the same advertiser, our method can always give the optimal solution. We also compare against two baseline methods:

(i) Grid search: search all available bid price in observations for each campaign, and then perform a search over all possible bid combinations from different campaigns before locating the one with the highest clicks within the budget limit. However, the major issue is combinatorial explosion.

(ii) Naive search: split the budget across different campaigns evenly. Given the diversified performances of different campaigns,
Table 1: An advertiser (id: 928285) with three campaigns given budget limit 100 . The optimal solution (optimal bid, optimal click, optimal spend) derived using ours, Grid search and naive method.

\begin{tabular}{c|l|l|l}
\hline \hline Method & optimal bid & optimal click & $\begin{array}{l}\text { optimal } \\
\text { spend }\end{array}$ \\
\hline Ours & {$[0.3127,0.4398,1 \mathrm{e}-9]$} & 638.5023 & 99.9891 \\
\hline Grid search & {$[0.3150,0.4400,0]$} & 625.1631 & 96.5623 \\
\hline Naive & {$[0.1641, \quad 0.6042$,} & 552.2347 & 100 \\
& $0.1597]$ & & \\
\hline \hline
\end{tabular}

Table 2: An advertiser (id: 1502906) with three campaigns given budget limit 5000 . The optimal solution (optimal bid, optimal click, optimal spend) derived using ours, Grid search and naive method.

\begin{tabular}{c|l|l|l}
\hline \hline Method & optimal bid & optimal click & $\begin{array}{l}\text { optimal } \\
\text { spend }\end{array}$ \\
\hline Ours & {$[\mathrm{e}-30,0.1823,0.2847]$} & 247616.17 & 5000 \\
\hline Grid search & {$[0,0.1850,0.2850]$} & 237935.89 & 4968.87 \\
\hline Naive & {$[0.023, \quad 0.1347$,} & 53776.80 & 5000 \\
& $0.2022]$ & & \\
\hline \hline
\end{tabular}

this approach, however, generally cannot produce the optimal solution.

Tables 1, 2 show the optimization results using two advertiser examples, where each advertiser has three different campaigns but with different performances of each campaign. We observe that our method always gives the best optimal solution. The same conclusion holds for other advertisers ${ }^{4}$.

\section{CONCLUSION}

This paper presents a novel way for portfolio bid optimization. The approach is novel in providing the optimal bid solution based on maximizing the clicks by splitting the budget across different campaigns for an advertiser. We also derive an effective method for obtaining the optimal solution with demonstrated advantages.

\section{REFERENCES}

[1] Han Cai, Kan Ren, Weinan Zhang, Kleanthis Malialis, Jun Wang, Yong Yu, and Defeng Guo. 2017. Real-Time Bidding by Reinforcement Learning in Display Advertising. CoRR abs/1701.02490 (2017).

[2] Ye Chen, Pavel Berkhin, Bo Anderson, and Nikhil R. Devanur. 2011. Real-time Bidding Algorithms for Performance-based Display Ad Allocation. In KDD'11. ACM, 1307-1315.

[3] Hongchang Gao, Deguang Kong, Miao Lu, Xiao Bai, and Jian Yang. 2018. ContextAware Attention Convolutional Neural Network for Advertiser-Level CTR Forecasting. In WWW'2018. ACM, to appear.

[4] Deguang Kong, , Konstantin Shmakov, and Jian Yang. 2018. An Inflection Point Approach for Advertising Budget Allocation. In WWW'2018. ACM, to appear.

[5] Deguang Kong, Konstantin Shmakov, and Jian Yang. 2018. Demystifying Advertising Campaign for CPA Goal Optimization. In WWW'2018. ACM, to appear.

[6] Harry Markowitz. 1952. Portfolio Selection. The fournal of Finance 7, 1 (1952), 77-91.

[7] Claudia Perlich, Brian Dalessandro, Rod Hook, Ori Stitelman, Troy Raeder, and Foster Provost. 2012. Bid Optimizing and Inventory Scoring in Targeted Online Advertising. In KDD'12. ACM, 804-812.

[8] Theodoros Tsagaris, Ajay Jasra, and Niall Adams. 2012. Robust and adaptive algorithms for online portfolio selection. Quantitative Finance 12, 11 (2012), 16511662 .

${ }^{4}$ Due to space limit, we can not present detailed advertiser result here. 